\title{
The biofilm produced by Burkholderia cepacia complex: molecular aspects and relationship with exopolysaccharides
}

\author{
Lucia Corich, Linda Furlanis, Lucilla Dolzani, Enrico Angelo Tonin, Cristina Lagatolla \\ Department of Life Science, University of Trieste
}

Key words: Biofilm, Exopolysaccharide, Cepacian, Burkholderia cepacia complex

II biofilm prodotto da Burkholderia cepacia complex: aspetti molecolari e relazione con gli esopolisaccaridi

\section{SUMMARY}

Introduction. In cystic fibrosis patients, Burkholderia cepacia complex $(\mathrm{Bcc})$ can cause serious pulmonary chronic infections thanks in part to the ability to form biofilm, matrix rich in exopolysaccharides. In Bcc grown in the planktonic state, the main exopolysaccharide is cepacian while in biofilm its presence is controversial.

Methods and Results. Two clinical isolates, named BTS7 and BTS2, were studied.

BTS7 produces abundant cepacian but not much biofilm (quantified by colorimetric method). At least two of the genes involved in cepacian biosynthesis are not necessary for biofilm production as two BTS7 derivatives, bceB and bceQ knocked out by transposon mutagenesis, produce biofilm levels comparable to the wild-type.

BTS2 sinthesyzes cepacian only if cultured on a specific medium. It has been colonizing a patient for almost ten years, showing a significant reduction of biofilm production during this period. This reduction did not appear together with the lack of factors required for the initial adhesion to the surface, or to differences in some of the Bcc genes involved in biofilm formation.

Moreover, sequencing of its bce locus revealed a bceX gene, absent in BTS7, coding for a trascriptional regulator. Its product may negatively regulate the production of cepacian but not the one of other polysaccharides, promoting the formation of biofilm.

Conclusions. Cepacian seems to be marginal in the production of biofilm. The reduced ability to produce biofilm of BTS2 suggests possible gene mutations occurred over time. Using custom arrays we will compare the gene expression of the BTS2 isolates, to identify the genes responsible for the observed phenotypic changes.

\section{INTRODUZIONE}

Nel polmone di pazienti affetti da fibrosi cistica, batteri appartenenti al Burkholderia cepacia complex (Bcc) possono causare gravi infezioni croniche (1) grazie anche alla capacità di formare biofilm, matrice ricca di esopolisaccaridi. Studi compiuti su Bcc cresciuto allo stato planctonico hanno messo in evidenza che l'esopolisaccaride prodotto più frequentemente è il cepaciano (2) (Figura I), ma nulla si sa riguardo alla sua presenza all'interno del biofilm.

\section{MATERIALI E METODI}

\section{Ceppi clinici di Bec analizzati}

Sono stati presi in considerazione due isolati clinici, responsabili di infezioni respiratorie croniche in pazienti affetti da fibrosi cistica (FC), differenti per il tipo di esopolisaccaridi (EPS) prodotto dalle forme planctoniche.

- BTS7: produttore esclusivamente di cepaciano, indipendentemente dal terreno di coltura.

- BTS7B \& BTS7Q: derivati di BTS7 incapaci di produrre cepaciano, ottenuti mediante transposon mutagenesis.

- BTS2: produttore di cepaciano ma anche di EPS differenti, a seconda del terreno di coltura.

I ceppi sono stati pre-incubati in due differenti terreni, Yeast Extract Mannitol (YEM) Medium e KING-A (KA), per indurre la produzione di diversi EPS.

\section{Valutazione in vitro della produzione di biofilm}

Il biofilm prodotto è stato quantificato come descritto (3), con l'introduzione di alcune modifiche: $5 \times 10^{5}$ batteri sono stati inoculati nei pozzetti di una piastra microtiter e incubati per 24 ore a $30^{\circ} \mathrm{C}$. Dopo aver misurato il livello di crescita $\left(\mathrm{OD}_{590}\right)$, le colture sono state lavate per eliminare le cellule non adese e fissate con metanolo. Il biofilm è stato colorato con cristalvioletto $2 \%$, decolorato con acido acetico glaciale $33 \%$ e quantificato mediante lettura spettrofotometrica $\left(\mathrm{OD}_{570}\right)$ del colorante estratto. Sono stati effettuati almeno tre esperimenti indipendenti, ciascuno eseguito in triplicato. I risultati sono espressi come Biofilm Index (BI), calcolato secondo la formula: $\left(\mathrm{OD}_{570}\right.$ Biofilm/ $/ \mathrm{OD}_{590}$ crescita batterica)*100 (4). Le differenze di BI sono state analizzate mediante Test $\mathrm{t}$ di Student; un valore di $p<0.05$ è stato considerato statisticamente significativo.

\section{RISULTATI}

La capacità di produrre biofilm da parte dei ceppi considerati si può riassumere nei seguenti punti:

- BTS7: produce biofilm in quantità piuttosto scarsa, paragonabile a quella prodotta dal ceppo di riferimento $B$. cenocepacia J2315 (Figura II A), che non produce cepaciano per una mutazione in uno dei geni essenziali.

- BTS7B \& BTS7Q: producono biofilm in quantità comparabili al ceppo selvaggio (Figura II B, C), suggerendo che almeno due dei geni coinvolti nella sintesi di cepaciano (bceB e bceQ) non sono necessari per la produzione del biofilm. Questi risultati fanno ipotizzare che la componente saccaridica prodotta all'interno del biofilm sia differente da quella rilasciata dalle forme planctoniche e i risultati preliminari ottenuti da un gruppo di biochimici con cui collaboriamo, secondo cui il polisaccaride prevalente all'interno del biofilm di Bcc sembra essere l'arabinogalattano mentre il cepaciano non risulta rilevabile, confermerebbero tale ipotesi.

- BTS2: produttore di EPS diversi a seconda del terreno di coltura utilizzato, è risultato capace di produrre biofilm in quantità molto abbondante (Figura II D). Poiché l'analisi di sequenza della regione adiacente al bceI locus ha rivelato in BTS2 la presenza di un gene regolatore di trascrizione, bceX, assente in BTS7 (Figura III), si potrebbe ipotizzare che tale gene regoli negativamente la produzione di cepaciano rispetto a quella di altri polisaccaridi.

Inoltre: 1'isolato BTS2, che colonizza un paziente da circa dieci anni, mostra una significativa riduzione della capacità di produrre biofilm a distanza di 6 (BTS2-06) e 9 (BTS2-09) anni rispetto al primo isolamento (BTS2-00) (Figura II D).

Allo stato attuale non sappiamo quali modificazioni geniche siano intervenute: le indagini preliminari a carico di alcuni geni coinvolti nella sintesi del biofilm non hanno evidenziato mutazioni tra il primo e l'ultimo prelievo.

\section{Corresponding author: Corich Lucia}

Università degli Studi di Trieste, Dipartimento di Scienze della Vita

Via L. Giorgieri, I - 34I00 Trieste - Tel.: 0405583693 - Fax: $040558369 \mid$

E-mail: luxcor@yahoo.it 


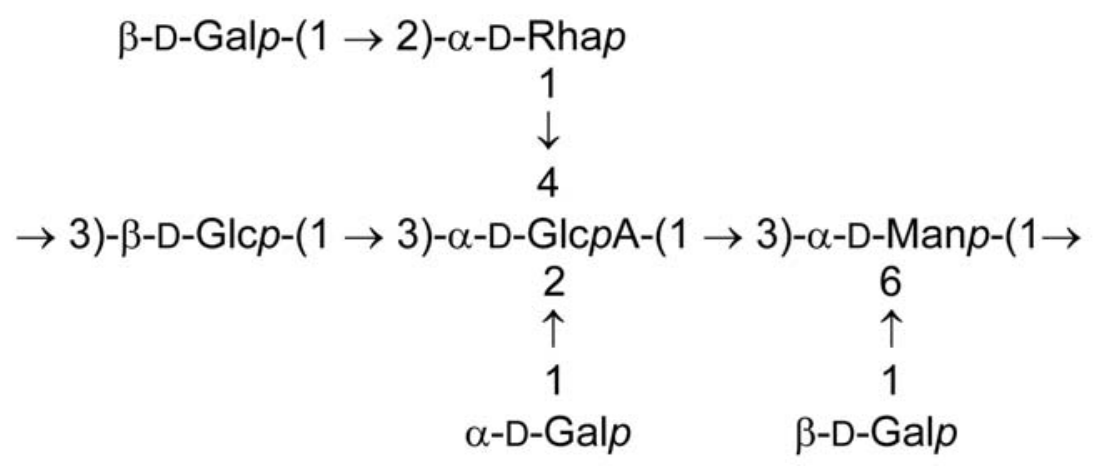

Figura I. Struttura dell'unità ripetitiva dell'esopolisaccaride cepaciano.

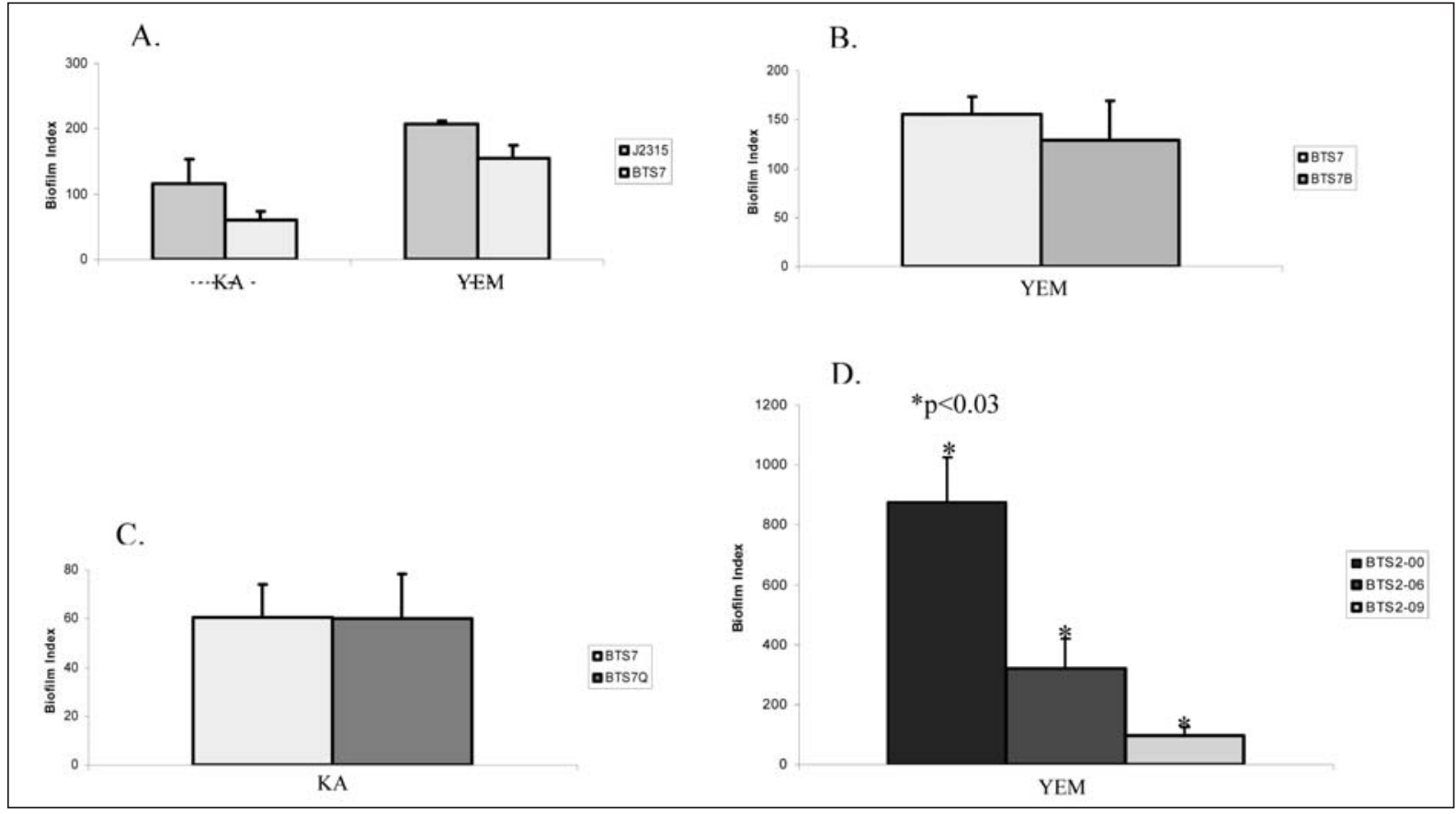

Figura II. Biofilm Index di isolati clinici di Bcc dopo 24 ore di incubazione in due diversi terreni:YEM (Yeast Extract Mannitol) e KA (KING-A).

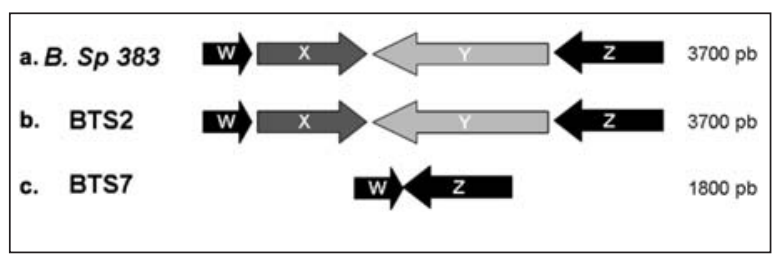

Figure III. Struttura dei geni adiacenti al bcel locus nel ceppo di riferimento Burkholderia spp. 383 (a) e negli isolati BTS2 (b) e BTS7 (c).

\section{DISCUSSIONE}

I risultati ottenuti dimostrano che il cepaciano, spesso prodotto dalle forme planctoniche di Bcc, riveste un ruolo marginale nella produzione del biofilm. Rimane però ancora da chiarire quali siano i meccanismi che regolano la produzione degli EPS in Bcc e, soprattutto, se la produzione di cepaciano piuttosto che di un altro EPS possa effettivamente favorire la crescita batterica in forma planctonica rispetto a quella sessile. Per verificare questa ipotesi prevediamo di sviluppare i nostri studi futuri secondo due linee di ricerca. Da un lato studieremo in maniera approfondita la funzione del gene $b c e X$, per verificare se la proteina codificata sia effettivamente in grado di modificare la produzione di cepaciano e/o del biofilm. Parallelamente, prendendo spunto dall'osservazione che ceppi responsabili di infezioni croniche vanno incontro a modificazioni fenotipiche durante la colonizzazione del polmone FC, prevediamo di confrontare, mediante custom array, l'espressione genica totale di isolati successivi del ceppo BTS2, per identificare i geni responsabili della modificazione fenotipica osservata.

\section{BIBLIOGRAFIA}

1. Govan JRW, Hughes JE, Vandamme P. Burkholderia cepacia: medical, taxonomic an ecological issues. J Med Microbiol 1996; 45: 395-407.

2. Richau J, Leitão JH, Correia M, et al. Molecular typing and exopolysaccharide biosynthesis of Burkholderia cepacia isolates from a Portuguese cystic fibrosis center. J Clin Microbiol 2000; 38: 1651-5.

3. Stepanovic S, Vukovic D, Hola V, et al. Quantification of biofilm in microtiter plates: overview of testing conditions and practical recommendations for assessment of biofilm production by staphylococci. APMIS 2007; 115 (8): 891-9.

4. Savoia D, Zucca M. Clinical and environmental Burkholderia strains: biofilm production and intracellular survival. Current Microbiol 2007; 54: $440-4$. 